\title{
SYSTEMATIC BUNDLE ADJUSTMENT OF HRSC IMAGE DATA
}

\author{
J. Bostelmann*, R. Schmidt, C. Heipke \\ Institute of Photogrammetry and GeoInformation (IPI) \\ Leibniz Universität Hannover \\ Nienburger Str.1, D-30167 Hannover, Germany \\ bostelmann@ipi.uni-hannover.de
}

\section{Commission IV, WG IV/7}

KEY WORDS: Extra-terrestrial, Mapping, Planetary, Matching, Bundle, Adjustment, Global, Analysis

\begin{abstract}
:
The European Mars Express mission was launched in June 2003 and sent into orbit around Mars. On board the orbiter is the German High Resolution Stereo Camera (HRSC). This multi-line sensor images the Martian surface with a resolution of up to $12 \mathrm{~m}$ per pixel in three dimensions and provides RGB and infra-red color information. The usage of the stereoscopic image information for the improvement of the observed position and attitude information via bundle adjustment is important to derive high quality 3D surface models, color orthoimages and other data products. In many cases overlapping image strips of different orbits can be used to form photogrammetric blocks, thus allowing the simultaneous adjustment of the exterior orientation data. This reduces not only local, but also regional inconsistencies in the data. With the growing number of HRSC image strips in this ongoing mission, the size and complexity of potential blocks is increasing. Therefore, a workflow has been built up for the systematic improvement of the exterior orientation using single orbit strips and regional blocks. For a successful bundle adjustment of blocks using multiple image strips a sufficient number of tie points in the overlapping area is needed. The number of tie points depends mainly on the geometric and radiometric quality of the images. This is considered by detailed analysis of the tie point accuracy and distribution. The combination of methods for image pre-processing, tie point matching, bundle adjustment and evaluation of the results in an automated workflow allows for all HRSC images a global assessment of the quality and a systematic selection of data for larger blocks.
\end{abstract}

\section{INTRODUCTION}

The Mars Express mission conducted by the European Space Agency (ESA) is investigating the Red Planet since January 2004. One of the scientific instruments on board the orbiter is the High Resolution Stereo Camera (HRSC). This multi-line stereo scanner is equipped with 5 panchromatic and 4 color charge-coupled device (CCD) line detectors, each with 5176 pixels. The configuration of the sensor lines on the focal plane of the camera allows a stereoscopic image acquisition of the Martian surface (Neukum et al., 2004). Until March 2012 the HRSC has orbited the planet 10,500 times aiming at a global coverage of image data.

By simultaneously reading out (up to) nine CCD sensor lines, while the camera points at Mars when reaching the periapsis of the highly elliptical orbit, long image strips are created. Because of the different viewing angles in the images of these strips, it is possible to obtain stereo color data, with a resolution of up to $12 m$ per pixel, depending on the flying height.

To use the HRSC image data for the derivation of digital terrain models (DTMs) or orthoimages the knowledge about the position and attitude of the camera is essential. The position is observed via Doppler measurements, and star trackers control the attitude of the camera. The combination of these observations is called the nominal exterior orientation of the camera. In many cases the quality of the exterior orientation is not accurate enough for a precise photogrammetric point determination. Therefore, a large number of automatically determined tie points is used in a bundle adjustment to improve the accuracy of the exterior orientation and to exploit the full potential of the stereo image data.

In many cases overlapping image strips of different orbits can be used to form photogrammetric blocks (see Fig. 1), thus allowing a more consistent adjustment of the exterior orientation data.

\footnotetext{
${ }^{*}$ Corresponding author
}

With the growing number of HRSC image strips in this ongoing mission, the size and complexity of potential blocks is increasing and a detailed knowledge about the photogrammetric capability of the HRSC data is needed to assure geometrically stable blocks.

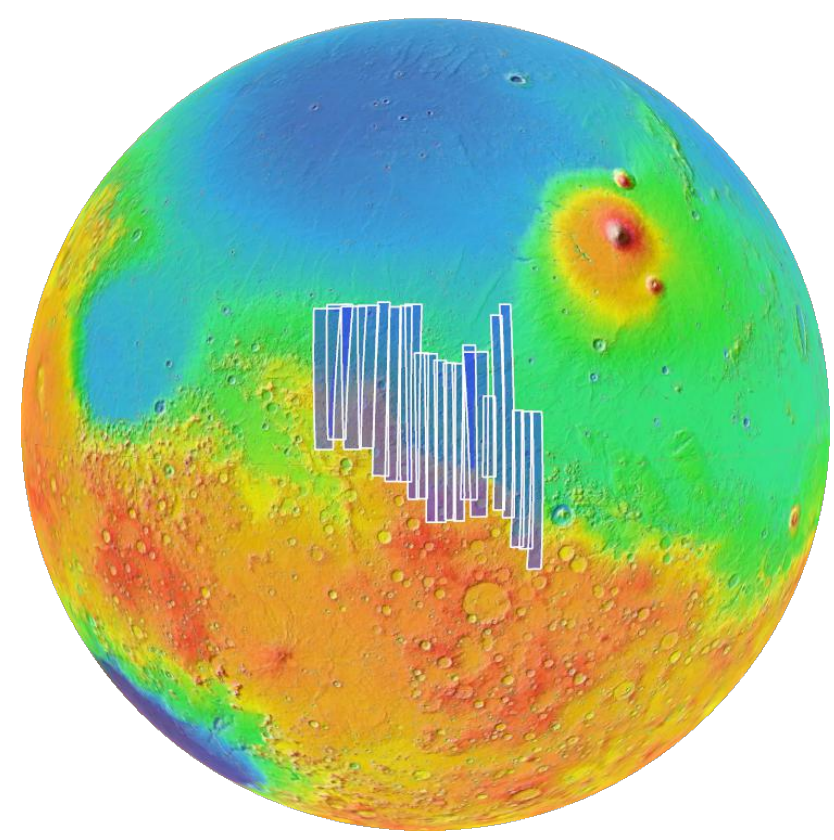

Figure 1: Color coded MOLA DTM with footprints of 21 HRSC nadir channel images constituting a photogrammetric block

We have developed a workflow for a systematic bundle adjustment of the HRSC image data. This workflow for single orbit strips is described in chapter 2 . In chapter 3 the extension of this method for multi orbit blocks is described. Results including a 
large block comprising 21 strips are presented in chapter 4 . The paper concludes with some observations for future work.

\section{SINGLE ORBIT STRIPS}

The workflow for a systematic bundle adjustment of the HRSC image data can be divided into four parts: image pre-processing, tie point matching, bundle adjustment and evaluation. In addition to the HRSC images, the nominal exterior orientation and the Mars Orbiter Laser Altimeter (MOLA) DTM are used as input. The adjusted exterior orientation is the output (see Fig. 2). The use of standard parameters for each processing step allows to apply this workflow to the entire HRSC data set without manual intervention. The most relevant parameters are described in the following sections.

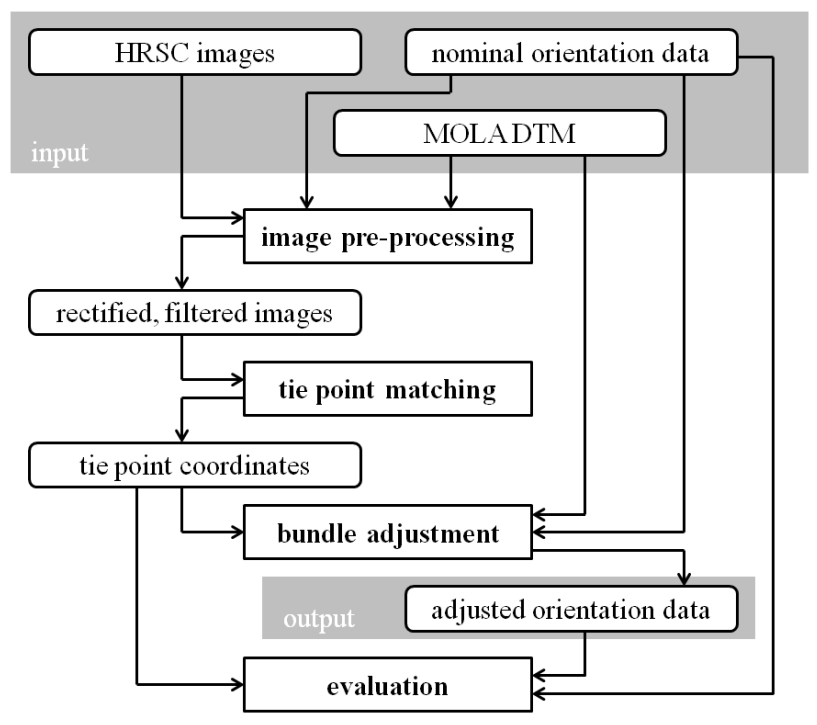

Figure 2: Workflow for systematic bundle adjustment of HRSC image data

\subsection{Image pre-processing}

The first step for the processing of single orbit strips is image pre-processing. In this step only the panchromatic channels of the HRSC images are used. Initially a low pass filter is applied to reduce image noise. A simple Gaussian with a $3 \times 3$ pixel filter matrix have shown good results and leads to a more robust tie point matching (Schmidt, 2008).

Subsequently the nominal orientation data and the MOLA DTM are used for a pre-rectification of the image data (Scholten et al., 2005). This step compensates scale differences and corrects nonquadratic pixels.

\subsection{Tie point matching}

In the nadir image the tie points are arranged as a regular grid. Given the desired number of points (approx. 45.000) and the image size a corresponding grid width is calculated. In contrast to an interest operator, the grid ensures a more regular distribution of the tie points. Tie point matching is carried out in the pre-rectified images. Depending on the image quality, the final number of successfully matched tie points lies between 20.000 and 45.000 .

Each candidate point in the nadir channel image is matched with the other four panchromatic channels. Because of higher macro pixel size and consequently a lower resolution, the color channels are normally not used for photogrammetric processing.
For image matching a pyramidal approach is used to take large parallaxes and imprecise nominal orientation values into account. As similarity measure a normalized cross correlation coefficient is employed. A $35 \times 35$ pixel correlation window is used. Subsequently the matching results are optimized by multi-image least squares matching (Schmidt et al., 2008).

\subsection{Bundle adjustment}

In the next part of the processing chain a combined bundle adjustment is carried out using the image coordinates of the tie points, the MOLA DTM and the nominal exterior orientation values (Spiegel, 2007a).

The used bundle adjustment method is based on the well known approach used in photogrammetry, by which the exterior orientation is simultaneously determined for all images. In case of HRSC the images are not acquired from individual view points but in a continuous motion. Thus, the exterior orientation must be modeled along the spacecrafts trajectory as a function of time. Three decades ago the concept of orientation points was proposed to solve this problem (Hofmann et al., 1982). Today, it is a common approach in the processing of multi-line sensor data.

To fit the photogrammetrically determined $3 \mathrm{D}$ coordinates of a photogrammetric bundle block to a regional or global reference system, an adequate number of ground control points is normally used in aerial or spaceborne photogrammetry. For areas without ground control points available DTMs can be used to obtain an absolute fit (Strunz, 1993). On Mars the MOLA DTM provides the best global accuracy. Therefore, a combined bundle adjustment for HRSC image data and the MOLA DTM as control information was developed, implemented and tested (Spiegel, 2007b).

The general approach of the bundle adjustment is a nonlinear least-squares adjustment. This optimization aims to find the best set of unknown model parameters to explain the observations. For a combined adjustment of HRSC images and the MOLA DTM there are four types of observations: image coordinates, orientation parameters, unknown bias and drift parameters to compensate systematic effects and DTM information. The four types of observation equations used in the bundle adjustment are described in (Bostelmann and Heipke, 2011).

The stochastic model of a bundle adjustment can be modified by changing the a priori standard deviation of a particular type of observation. This allows to handle observations as constants with $\sigma_{0}=0$, as free unknowns with $\sigma_{0} \rightarrow \infty$ or as stochastic values with a $\sigma_{0}$ of something in between. The strategy specially developed for HRSC data divides the bundle adjustment into two parts with different values for the stochastic model (Spiegel, 2007b). The image coordinates of the tie points are always introduced with an a priori standard deviation of $\sigma_{0, x y}=1 \mu \mathrm{m}$.

In the first part only the nominal orientation parameters at the orientation points and the tie points are introduced, but no drift and bias and no DTM information. The a priori standard deviation for the orientation is chosen as $\sigma_{0, \varphi \omega \kappa}=0.028 \mathrm{gon}$ for the attitude and $\sigma_{0, X Y Z}=0.01 \mathrm{~m}$ for the position. The latter considers the stable orbit of the spacecraft. This part optimizes the internal accuracy of the strip.

In the second part observations for bias and drift are introduced with $\sigma_{0, b i a s, X Y Z}=1000 \mathrm{~m}$ allowing a translation of the whole strip. A drift for the height component of the exterior orientation is permitted by setting $\sigma_{0, d r i f t, Z}=0.01 \mathrm{~m} /$ imageline. For $\mathrm{X}$ and $\mathrm{Y}$ no drift is introduced. For the orientation angles neither 
bias nor drift are considered. This modification of the stochastic model allows to fit the HRSC object points to the MOLA DTM, which is introduced with $\sigma_{0, D T M}=100 \mathrm{~m}$ for the terrain points. In this part the absolute position of the image strip is adjusted in an iterative way.

Both parts use blunder detection. In the first part blunders in the tie points are detected iteratively by analyzing the residuals. In the second part the difference between the MOLA surface and the HRSC points is used to find non-fitting points (Spiegel, 2007b).

\subsection{Evaluation}

To evaluate the performance of the bundle adjustment, the quality of the nominal and adjusted exterior orientation data is compared. Because of the large amount of data, a single value describing the geometric quality is easier to handle for systematic analysis of the whole data set. The mean forward ray intersection error summarizes the accuracy of all object points and is a good measure for the internal consistency of the data (Gwinner et al., 2009). The evaluation step calculates the forward intersection error for all object points twice. Both times it uses the same set of tie point coordinates and only switches between nominal and adjusted orientation data, so that the resulting mean intersection error reveals the gain in quality.

Additionally, both the nominal and the adjusted orientation information is used to generate dense 3D point clouds as used for DTM generation (Gwinner et al., 2010). The intersection error for all these points is used to plot two color coded error maps. This allows a visual inspection of the spatial distribution and accuracy of the HRSC points. Fig. 3 shows three examples of error maps. Strip h7295_0000 displays large black areas where the dense matching algorithm was not able to find conjugate image points. The color of the small number of points, shows a low accuracy. There is no significant improvement through bundle adjustment. This is also indicated by the values for the mean intersection error $(38.6 \mathrm{~m}$ and $37.7 \mathrm{~m})$. In contrast, the error maps and mean intersection error of h2091_0000 and h8500_0000 show considerable improvement after bundle adjustment despite of the high frequent oscillations visible in strip h8500_0000.

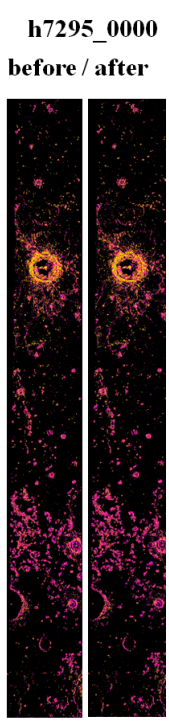

$38.6 m \quad 37.7 m$

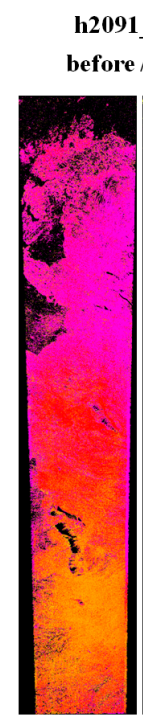

$46.3 \mathrm{~m}$

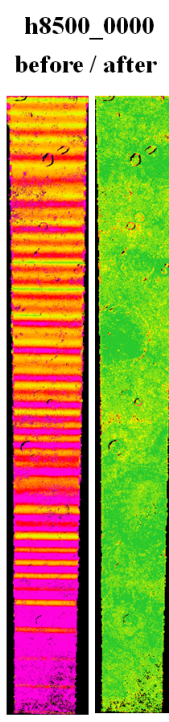

$44.3 \mathrm{~m} \quad 5.5 \mathrm{~m}$

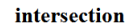

error
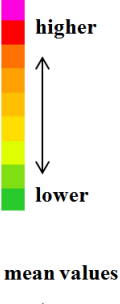

|
Figure 3: Error maps with mean intersection error of the object points (black: no points)

\section{MULTI ORBIT BLOCKS}

For the processing of photogrammetric blocks containing images from more than one HRSC strip an adaption of the same workflow for single orbit strips (Fig. 2) can be used (Spiegel, 2007b).

To efficiently process large image blocks a suitable strategy has to be set up. It is not reasonable to process all image strips of the entire block together because only neighboring image strips overlap. Therefore, the whole image block is broken down into smaller sub-blocks which additionally opens up the advantage of parallel processing. If a sufficient number of server nodes respectively CPU cores is available, it is possible to run three of the four processing parts (image pre-processing, tie point matching and evaluation) for all sub-blocks simultaneously. This drastically saves computation time.

For the tie point matching each sub-block needs a reference image which is matched against all other overlapping images. Every strip of the entire block has to supply this reference image once. If e.g. the block consists of two overlapping strips with ten single images in total the nadir channel of the first strip is matched against all other nine images. The second sub-block in this example would simply consist of the second strip. At this point merely the remaining area which was not captured by the first strip is covered.
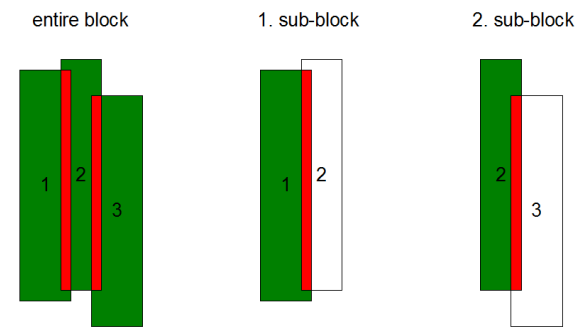

3. sub-block

Figure 4: Concept of sub-blocks using the example of three strips, green: 5-ray points, red: 10-ray points

This strategy on the basis of three overlapping strips is depicted in Fig. 4. Three sub-blocks are built up in the combinations $1 \& 2,2$ $\& 3$ and 3 separately. An advantage of this approach in particular with strips of inhomogeneous geometry is that for each sub-block an optimal average matching scale can be defined. In case of processing all image strips simultaneously an average matching scale for the complete block would have to be found.

Concerning computational limitations the number of tie points per sub-block has to be limited. Tests have shown that if a sufficient number of tie points in all overlapping areas is available, a further increase of the number of points does not improve the results. While it is clear that this strategy will fail for crossing strips as to be found e.g. on the poles it is good compromise with regard to computing power.

In the subsequent bundle block adjustment the results of the subblocks are imported and processed simultaneously. As approximate values of the exterior orientation for the pre-rectification of the images the results from single orbit strip processing are used. The reason is that while images of one strip normally exhibit a high relative accuracy, the discrepancy between neighboring image strips can reach several hundred meters which would require very large search spaces for matching. When employing adjusted image strips the orbits fit much better among each other so that smaller search spaces can be used.

The iterative blunder detection during bundle adjustment is conducted initially for all strips independently as decribed above, and 
afterwards also in the overlapping areas. Again, the blunders are detected firstly in ray intersections only, and secondly with referenc to the MOLA DTM (Spiegel, 2007b).

For the evaluation of the bundle adjustment results the mean intersection error is calculated only for point lying in an overlapping area. This reveals the improvement of the bundle adjustment of blocks more clearly than the usage of all points of a sub-block would do.

\section{RESULTS}

\subsection{Systematic bundle adjustment of single orbit strips}

The method for systematic bundle adjustment of single orbit strips was used to process the HRSC data received in the almost 7 years from January 2004 until November 2010. During this time the Mars Express mission has surrounded the planet 8761 times. In about 2800 of these orbits the camera took imagery theoretically suitable for stereo processing. HRSC image data sets of 2535 strips were succesfully processed. In 2329 strips (91.9\%) the exterior orientation was significantly improved by the bundle adjustment. For a number of 123 strips further improvements of the exterior orientation were achieved by modeling spacecraft oscillations through a shorter orientation point distance (Bostelmann and Heipke, 2011).

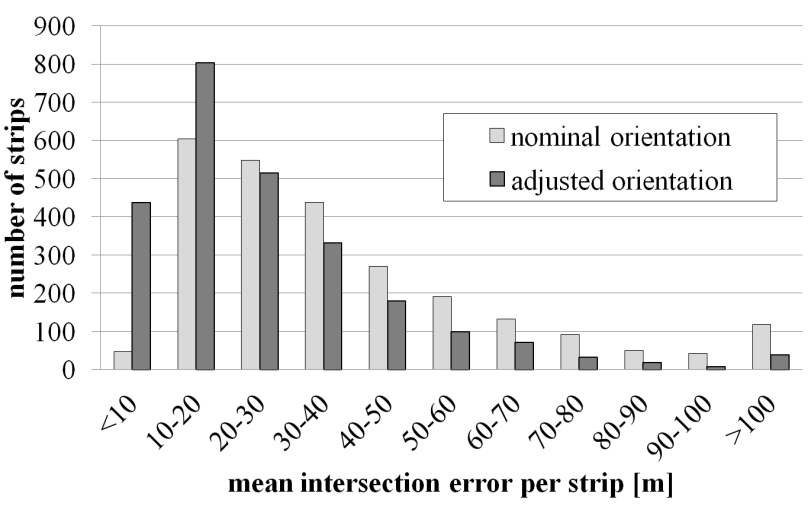

Figure 5: Number of strips grouped by their mean intersection error before (light gray) and after (dark gray) the improvement of the exterior orientation by bundle adjustment

In Fig. 5 the mean intersection error for a total of 2535 successfully processed strips is presented. The strips are classified according to their intersection error in ranges of $10 \mathrm{~m}$. The light gray bars represent the quality of the exterior orientation data before, and the dark gray bars after the process of bundle adjustment. It is shown that the number of strips with a mean intersection error $<10 \mathrm{~m}$ distinctly increases after the bundle adjustment (more precisely from 47 to 437 ). Also the quantity of strips with a mean intersection error lying between $10 \mathrm{~m}$ and $20 \mathrm{~m}$ increases by a number of 200 . On the other hand the number of strips with higher errors obviously decreases. E.g. the number with an error $>100 \mathrm{~m}$ is reduced from 119 to 38 strips. Probably because there was no exclusion of strips with obvious image degradation, the mean intersection errors presented here are somewhat higher than in other statistics of HRSC DTM processing (e.g. Gwinner et al., 2010). In general the mean intersection error presented in this paper should not be taken as an absolute criterion for the quality of HRSC data, but it is a good indicator for a relative improvement.

\subsection{Multi orbit block processing}

The analysis of single orbit strips reveals the internal photogrammetric accuracy of HRSC image data. To connect different strips to a stable block, in addition a sufficient number of accurate tie points in the overlapping area is needed. The previously described method of adjusting the exterior orientation of a block was used for a set of 21 overlapping HRSC strips. The selected area is a region called Nepenthes Mensae between $1^{\circ} \mathrm{S}$ to $17^{\circ} \mathrm{N}$ and $111^{\circ} \mathrm{E}$ to $135^{\circ} \mathrm{E}$. The footprints of the nadir channel images are shown as an overlay to the color coded MOLA DTM in Fig. 6.

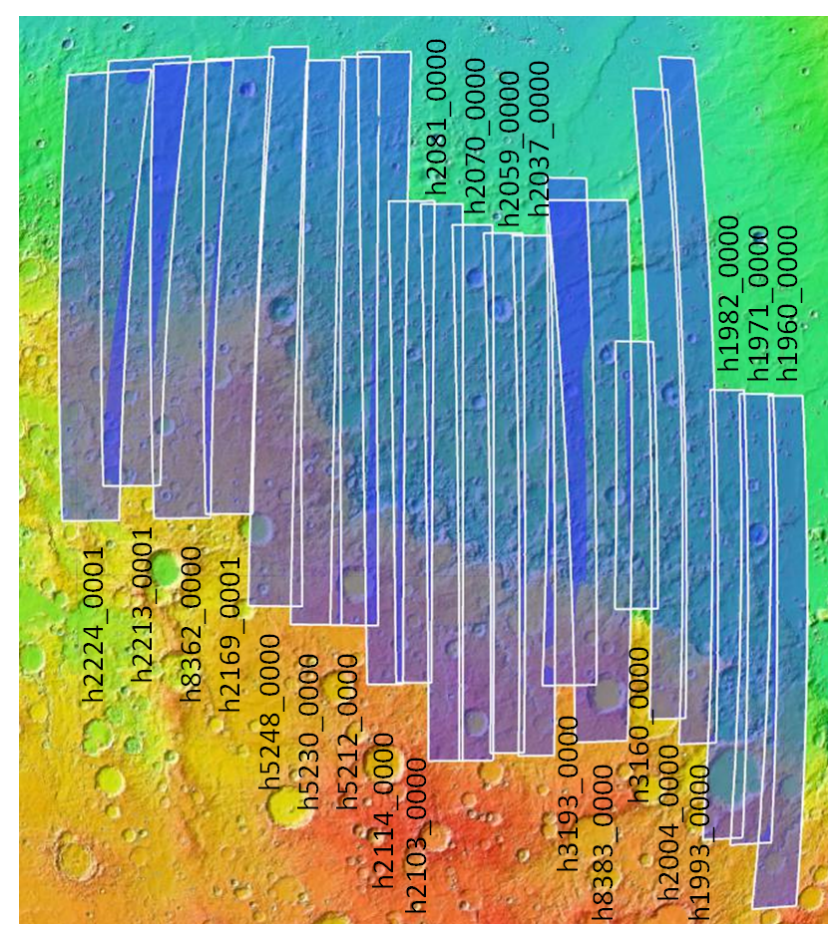

Figure 6: Color coded MOLA DTM with footprints of 21 HRSC nadir channel images constituting a photogrammetric block

The criteria for the selection of these strips were:

- The 21 strips overlap to form a single block.

- All five panchromatic channels are available.

- The single strip bundle adjustment succeeded with a small mean intersection error (less than $20 \mathrm{~m}$ ).

- The resolution of the nadir channel is better than $30 \mathrm{~m}$.

- The image information allows tie points distributed over the whole image.

- The overlapping area between neighboring strips is relatively wide.

Table 1 lists the selected strips. They are ordered going from west to east. The data was aquired in four time frames. 14 of the strips were taken in the year 2005. Strip h3193 and h3160 were taken 9 months later in 2006. Three strips (h5212, h5230 and h5248) were taken in 2008. The newest two strips (h8362 and h8383) from 2010 fill the remaining gaps. Fig. 7 shows the mean intersection error of the strips before and after the single strip bundle adjustment. The use of nominal exterior orientation results in a mean intersection error of $30 \mathrm{~m}$ or higher in some strips. With the adjusted orientation data it is reduced for all strips to a mean of $11.3 \mathrm{~m}$. 


\begin{tabular}{|c|c|c|}
\hline HRSC Strip & Date & Nadir Resolution [m] \\
\hline \hline h2224_0001 & $2005-10-07$ & $20.9-31.6$ \\
\hline h2213_0001 & $2005-10-04$ & $20.5-30.4$ \\
\hline h8362_0000 & $2010-07-15$ & $19.8-29.2$ \\
\hline h2169_0001 & $2005-09-22$ & $16.3-24.3$ \\
\hline h5248_0000 & $2008-02-02$ & $13.7-18.7$ \\
\hline h5230_0000 & $2008-01-28$ & $13.3-17.5$ \\
\hline h5212_0000 & $2008-01-22$ & $13.1-16.3$ \\
\hline h2114_0000 & $2005-09-06$ & $12.2-18.7$ \\
\hline h2103_0000 & $2005-09-03$ & $12.0-15.7$ \\
\hline h2081_0000 & $2005-08-28$ & $11.6-14.4$ \\
\hline h2070_0000 & $2005-08-25$ & $11.6-13.7$ \\
\hline h2059_0000 & $2005-08-22$ & $11.6-13.2$ \\
\hline h2037_0000 & $2005-08-16$ & $12.2-12.7$ \\
\hline h3193_0000 & $2006-07-05$ & $13.8-18.5$ \\
\hline h8383_0000 & $2010-07-21$ & $18.3-28.2$ \\
\hline h3160_0000 & $2006-06-26$ & $13.2-15.1$ \\
\hline h2004_0000 & $2005-08-06$ & $12.8-12.6$ \\
\hline h1993_0000 & $2005-08-03$ & $13.4-12.5$ \\
\hline h1982_0000 & $2005-07-31$ & $14.8-12.1$ \\
\hline h1971_0000 & $2005-07-28$ & $15.5-12.4$ \\
\hline h1960_0000 & $2005-07-25$ & $17.2-12.6$ \\
\hline
\end{tabular}

Table 1: HRSC strips used for the block

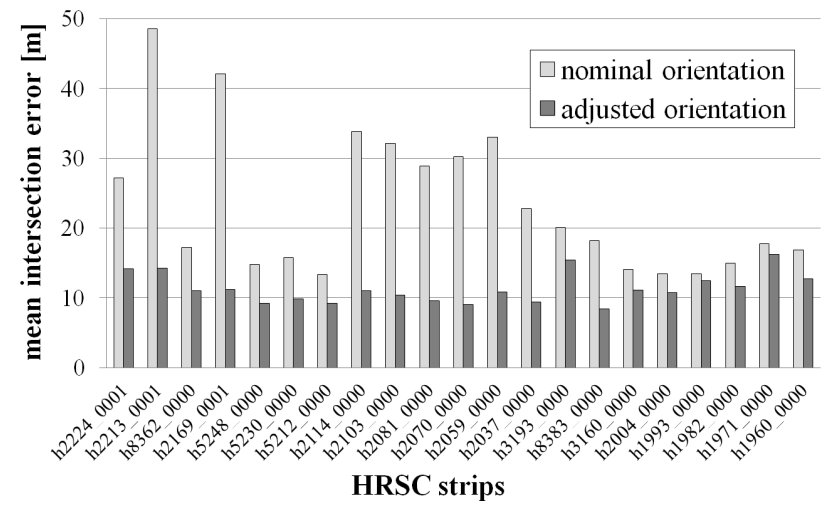

Figure 7: Mean intersection error before and after single strip bundle adjustment

For the stability of the complete block the spatial accuracy of tie points in the overlapping areas is important. Hence for all $20 \mathrm{sub}-$ blocks with an overlapping area the mean intersection error was calculated using the different orientation data sets (Fig. 8).

For the computation of the complete block as a whole, the number of tie points for each sub-block was reduced to a target value of 8.000 .

In all but one sub-block the block adjusted orientation data show a clearly higher accuracy for the HRSC points in the overlapping area than orientation data adjusted for single strips. In sub-block $\mathrm{h} 2169-\mathrm{h} 5248$ the overlapping area is too small to find a sufficient number of tie points. All sub-blocks with a mean intersection error notably higher than $10 \mathrm{~m}$ consist of two image strips with different illumination conditions (Fig. 9).

Overall the adjustment of the exterior orientation in a regional block does not only provides results with a high internal accuracy for each strip, but also the relative orientation of the strips is clearly improved. Additionally the block as a whole is fitted to a global reference system, the MOLA DTM. The block adjusted orientation data are the most appropriate ones for the subsequent derivation of a DTM mosaic or the rectification of ortho-images

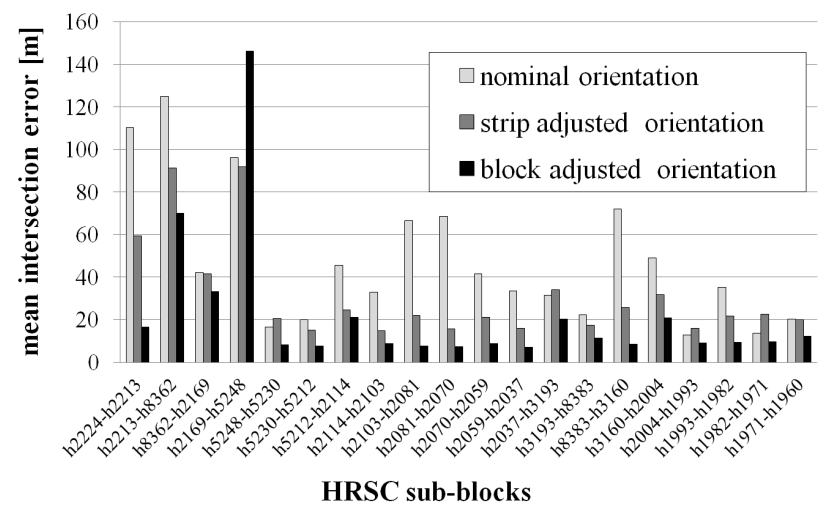

Figure 8: Mean intersection error for overlapping areas in subblocks calculated with different orientation values

(Dumke et al., 2008). For the depicted example of 21 image strips, the HRSC DTM mosaic is shown in Fig. 10.

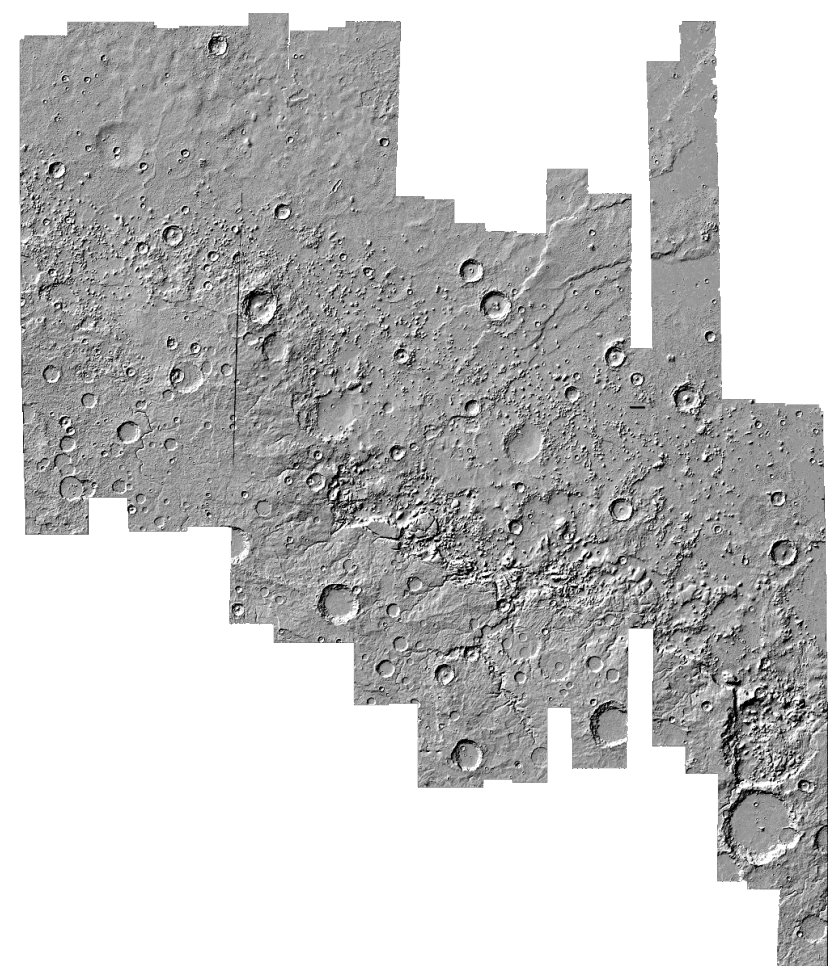

Figure 10: Shaded relief of a HRSC DTM mosaic generated with the block adjusted orientation data

\section{CONCLUSIONS AND FUTURE WORK}

The workflow for systematic bundle adjustment of HRSC image data was used to improve the exterior orientation of single strips and a regional block. It has been shown that for 2329 single strips a significant improvement was achieved. A block comprising 21 HRSC strips was simultaneously adjusted and the results were evaluated to demonstrate the stability of the overlapping areas.

The automated implementation of the workflow and the parallel processing using the performance of a university computing center allow to locate stable overlapping areas in all available HRSC strips in a systematic way. The results of this analysis can be used to design larger regional blocks. It is also possible to investigate blocks of strips connected to rings around the equator and if gaps in the HRSC data are filled with images from other missions a closed global block completely covering the Martian surface. 


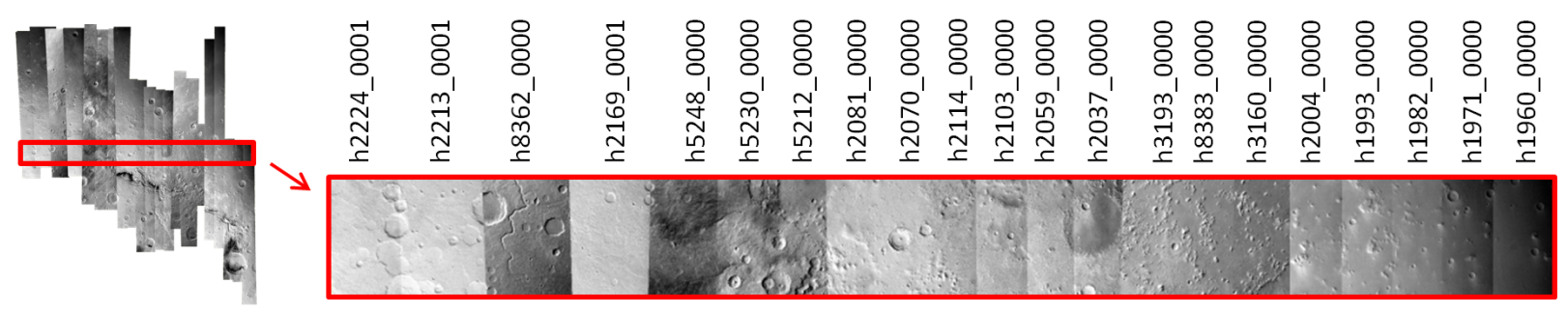

Figure 9: Illumination differences in the orthoimage mosaic rectified with the block adjusted orientation data and the HRSC DTM mosaic

\section{REFERENCES}

Bostelmann, J. and Heipke, C., 2011. Modeling spacecraft oscillations in HRSC images of Mars Express. International Archives of Photogrammetry and Remote Sensing, 38 (4/W19), Hannover.

Dumke, A., Spiegel, M., Schmidt, R., Michael, G. and Neukum, G., 2008. Mars: High resoluition digital terrain model and ortho-image mosaic on the basis of MEX/HRSC data. International Archives of Photogrammetry and Remote Sensing 37(4), pp. 1037-1042.

Gwinner, K., Scholten, F., Preusker, F., Elgner, S., Roatsch, T., Spiegel, M., Schmidt, R., Oberst, J., Jaumann, R. and Heipke, C., 2010. Topography of Mars from global mapping by HRSC highresolution digital terrain models and orthoimages: Characteristics and performance. Earth and Planetary Science Letters 294(3-4), pp. 506-519.

Gwinner, K., Scholten, F., Spiegel, M., Schmidt, R., Giese, B., Oberst, J., Heipke, C., Jaumann, R. and Neukum, G., 2009. Derivation and Validation of High-Resolution Digital Terrain Models from Mars Express HRSC-Data. Photogrammetric Engineering \& Remote Sensing 75(9), pp. 1127-1142.

Hofmann, O., Nave, P. and Ebner, H., 1982. DPS - A Digital Photogrammetric System for Producing Digital Elevation Models and Orthophotos by Means of Linear Array Scanner Imagery. International Archives of Photogrammetry and Remote Sensing 24 (3), pp. 216-227.

Neukum, G., Jaumann, R. and the HRSC Co-Investigator Team, 2004. HRSC: The High Resolution Stereo Camera of Mars express. Mars Express: The Scientific Payload, Eur. Space Agency Spec. Publ., ESA-SP 1240, pp. 17-36.

Schmidt, R., 2008. Automatische Bestimmung von Verknüpfungspunkten für HRSC-Bilder der Mars ExpressMission. Dissertation, DGK-C, Nr. 623, Deutsche Geodätische Kommission, München.

Schmidt, R., Spiegel, M., Heipke, C., Dumke, A., Neukum, G. and the HRSC Co-Investigator Team, 2008. Operational Determination of Tie Points and Bundle Adjustment of HRSC Images of the Mars Express Mission. International Archives of Photogrammetry and Remote Sensing 37(4), pp. 1025-1030.

Scholten, F., Gwinner, K., Roatsch, T., Matz, K., Wahlisch, M., Giese, B., Oberst, J., Jaumann, R. and Neukum, G., 2005. Mars Express HRSC Data Processing-Methods and Operational Aspects. Photogrammetric Engineering and Remote Sensing 71(10), pp. 1143-1152.

Spiegel, M., 2007a. Improvement of Interior and Exterior Orientation of the Three Line Camera HRSC with a Simultaneous Adjustment. International Archives of Photogrammetry and Remote Sensing 36(3/W49B), pp. 161-166.
Spiegel, M., 2007b. Kombinierte Ausgleichung der Mars Express HRSC Zeilenbilddaten und des Mars Global Surveyor MOLA DGM. Dissertationsschrift, DGK-C, Nr. 610, Deutsche Geodätische Kommission, München.

Strunz, G., 1993. Bildorientierung und Objektrekonstruktion mit Punkten, Linien und Flächen. Dissertationsschrift, DGK-C, Nr. 408, Deutsche Geodätische Kommission, München.

\section{ACKNOWLEDGEMENTS}

We thank the HRSC Experiment Teams at DLR Berlin and Freie Universität Berlin as well as the Mars Express Project Teams at ESTEC and ESOC for their successful planning and acquisition of data as well as for making the processed data available to the HRSC Team. This work is funded by the Deutsches Zentrum für Luft- und Raumfahrt e.V. (DLR) under grant no. 50 QM 0902. This support is gratefully acknowledged. 